\title{
Modified Proportional Fair Scheduling Algorithm for Heterogeneous LTE-A Networks
}

\author{
https://doi.org/10.3991/ijim.v14i10.14389 \\ Nada M. Elshennawy $(\bowtie)$ \\ Tanta University, Tanta, Egypt \\ Nada elshennawyef-eng.tanta.edu.eg
}

\begin{abstract}
Growing of time-sensitive applications such as streaming multimedia, voice over IP and online gaming required strongly support from mobile communication technology. So, the persistent need for wireless broadband technologies such as LTE-A is essential. LTE-A can achieve QoS in an efficient manner by using a reliable packet scheduling algorithm. It also supports good cell coverage by using heterogeneous capability. In this paper, modifications of proportional fair (PF) algorithm are proposed with different methods to compute the average throughput, which is the main and important parameter in the PF cost function. These methods are geometric mean, root mean square and arithmetic mean. Vienna simulator is used to study the performance of the proposed algorithms. A comparison between PF modifications and the most famous algorithms (the original PF and Best CQI algorithms) with various UE velocities is introduced. Average UE throughput, spectral efficiency, energy per bit, cell throughput and fairness are used as performance indicators. The results expose that QPF has best improved values for spectral efficiency, energy per bit and fairness by $8.4 \%, 14 \%$, and $9.3 \%$, respectively than original PF. However, Best CQI has a better value of average UE and cell throughput than all algorithms of $2 \%$ and $1.8 \%$ in low and medium UE velocity, respectively, but the best value of all types of throughput at high speed is gained by QPF. QPF and GMPF has the same performance in fairness with all UEs speeds.
\end{abstract}

Keywords-Long Term Evolution Advanced (LTE-A), Heterogeneous network (HetNet), QoS Scheduling Algorithms, Proportional Fair (PF), root mean square (RMS).

\section{Introduction}

Increasing in mobile applications needs special wireless communication technology which should have special features such as quality of services (QoS) support and high data rate. It is essential to develop a viable and powerful wireless technology. Long term evolution (LTE) is developed by the third Generation Partnership Project (3GPP) to introduce the mobile service requirements strongly. It also supports high data rates, low latency, better coverage and better QoS [1]. 3GPP is still developing its LTE to face the rapid growth in the multimedia applications and it has introduced an LTE improvement named LTE advanced (LTE-A) which provides users with better services, 
has ability of servicing users with better performance and an improved the overall network performance [2].

LTE-A has provided many advanced features: Carrier Aggregation (CA), Multipleinput and multiple-output (MIMO), Coordinated multipoint (CoMP) and Heterogeneous Networks (HetNets) [2]. In LTE-A, heterogeneous networks are presented by using many base stations with different levels of power to increase the coverage area [3].

In LTE-A, the main part which responses for allocating the resources fairly between users is the radio resources management (RRM). It is a very important aspect, especially in heterogeneous networks [4]. The distribution of the resource between the users is very critical and difficult process, and it should be done at each Transmission Time Interval (TTI). Each user has different types of applications; therefore, the network technology should be able to support different quality of service (QoS) needs. Because, the system performance indicators like throughput and fairness between users are affected by choosing the scheduling algorithm [4].RRM has two main tasks: Radio Admission Control (RAC) and Packet Scheduler (PS) which can be considered as the two wings of QoS. RAC has the capability of testing the network availability for new transmissions in both downlink and uplink before the acceptance of new users. PS has the responsibility of allocating the Resource Blocks (RBs) efficiently in TTI between users to improve the overall network performance [4].

The most important phase in QoS in LTE-A is the packet scheduling which allocates sub-carriers resources for User Equipment (UEs) in each TTI in a manner that maximizes the overall network performance [2]. LTE-A scheduling algorithms can be classified into: channel-aware and unaware algorithms. In channel-unaware algorithms, the algorithm takes parameters as throughput, delay, fairness and energy consumption in its priority function such as proportional fair $(\mathrm{PF})$ algorithm. But, in channel-aware, the channel status is the main parameter of its function such as Best Channel Quality Indicator (Best CQI) algorithm [4].PF is the most powerful, efficient and fair used algorithm in LTE-A, because it maximizes fairness with an acceptable values in the other performance parameters: average throughput, spectral efficiency and used energy [4]. In the literature, many modifications of PF are developed presenting different methods to calculate the average throughput value in its cost function.

In [5], the difficulties designing issues for downlink scheduling algorithms are introduced. To illustrate the importance of the relation between fairness and throughput. In [6], performance analysis between Open Loop Spatial Multiplexing (OLSM) and Closed Loop Spatial Multiplexing (CLSM), for PF and Round Robin (RR) algorithms with a variety of UE velocities for LTE-A heterogeneous networks are introduced. The results highlight that at low UEs velocity, CLSM-PF has best performance with the desired MIMO-scheduler combination. However, at high velocities, OLSM-RR is the preferred combination. Resource allocation study in Femto cell for LTE-A networks is reviewed in [7]. A resource allocation strategy is also suggested. It uses the Heterogeneous Channel Quality Index (CQI) based Scheduling Techniques (HCBST) with indexed adaptive modulation and coding technique by referring the various CQI parameters. Finally, the results show that HCBST system is better in term of throughput and spectral efficiency than the existing scheduling algorithms. 
In [8], a packet scheduling algorithm for the downlink LTE system is developed based on Kalman filter to restore the true CQI from erroneous channel quality feedback. Then, a time domain grouping of PF and Modified Largest Weighted Delay First (MLWDF) algorithms is also used. The results illustrate that the throughput and packet loss ratio of the proposal has better performance.In this paper, modifications on PF are developed, by using different methods to calculate the average throughput in its cost function. These methods are the root mean square, arithmetic mean, and geometric mean which were used in LTE-A homogenous networks in the literature $[9,10,11]$. In these methods. Proposed algorithms performance is studied by comparing their performance with the original PF and Best CQI algorithms at various UE velocities. Average throughput for UE and cell, fairness, average used energy per bit and spectral efficiency are used as performance indicators.

The paper is organized as follows. In Section II, an overview of LTE-Advanced and its heterogeneous type is introduced. Section III describes the scheduling algorithms. In Section IV, the proposed modifications are explained. The simulation results and discussion are reviewed in Section V. Finally, conclusion and future works are presented in Section VI.

\section{LTE-A and Heterogeneous Networks}

LTE-A stands for long term evolution-advanced. It is developed by 3rd Generation Partnership Project (3GPP) as a viable wireless technology. LTE-A architecture is shown in fig. 1. As declared from the figure, the two main parts of LTE-A: E-UTRAN (Evolved Universal Terrestrial Radio Access Network), also called base stations, (eNBs) and Evolved Packet Core (EPC) [1].

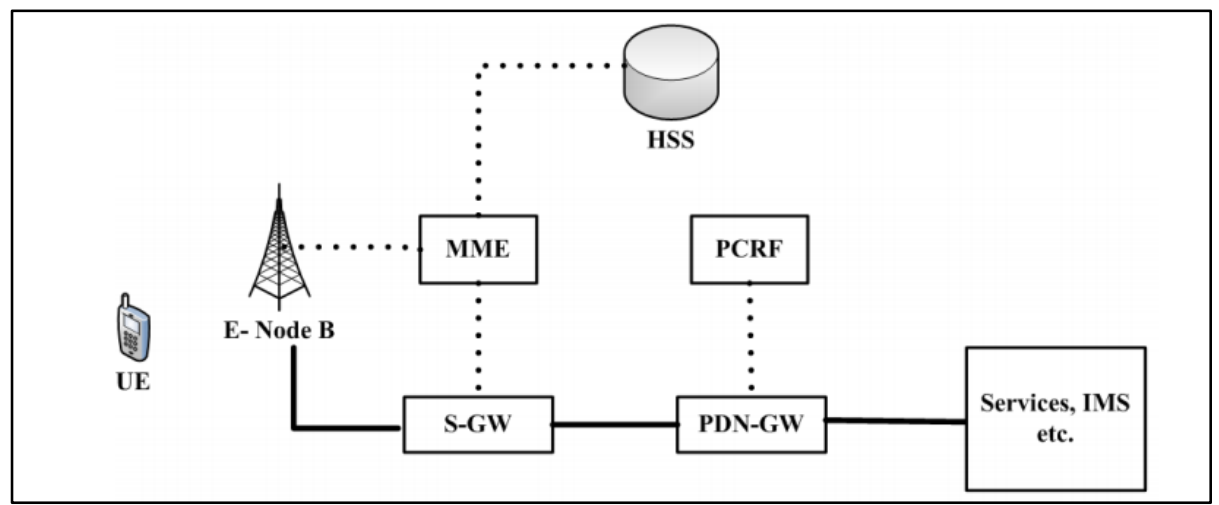

Fig. 1. LTE-A Architecture

LTE-A uses in its physical layer, OFDMA (the Orthogonal Frequency Division Multiple Access) for downlink (DL) and Single Carrier FDMA for uplink (UL) [12, 13]. It also has many features like: achieving a high level of data rate, supporting low latency for real time applications, using Multiple Input Multiple Output (MIMO), Carrier 
Aggregation (CA), Coordinated Multipoint (Comp), and finally, introducing strong coverage in its cell by using Heterogeneous Network (Het Net).

Wireless network layout is classified into [14]: homogeneous networks and heterogeneous network. All used base-stations have the same: power transmission level, antenna patterns, receiver noise floors, and similar backhaul connectivity to the data network, in homogeneous networks. Also, base stations have unrestricted assess to user terminals and support different applications with various QoS requirements. Their locations are chosen to maximize the coverage and control the interference between them.

Heterogeneous networks have many types of base stations based on the power levels to improve the network coverage area as shown in fig. 2 . This type of networks consists of a regular macro base-stations which have high power transmission level ( $\sim 5 \mathrm{~W}-40$ W), overlaid with several pico stations, femto base-stations and relay base-stations, which have transmission power at lower levels $(\sim 100 \mathrm{~mW}-2 \mathrm{~W})[14]$.

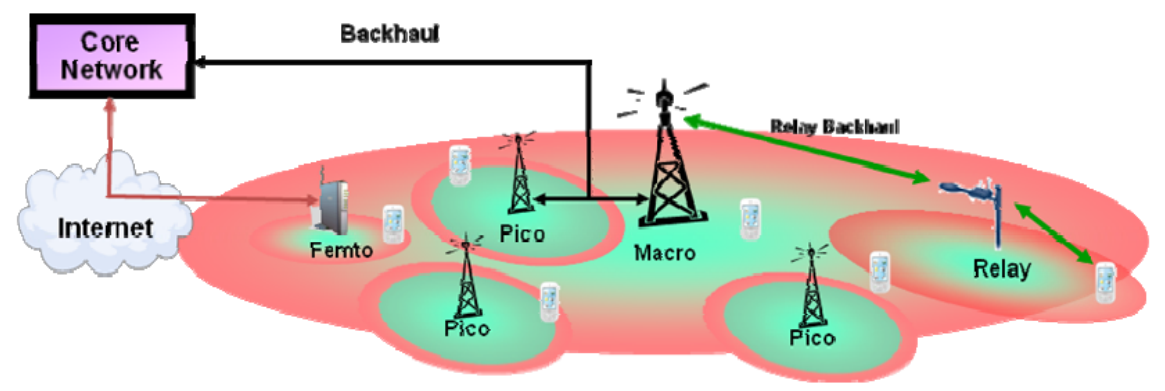

Fig. 2. Heterogenous Networks [13]

QoS in LTE-A can be considered as a main and important concept. The hardest challenge in wireless communication technologies is supporting strong QoS to satisfy different user requirements and applications over the network. Scheduling algorithm is one of the principal methods used to preserve QoS which prioritizes applications in the network based on the service type, as each application requires special characteristics like delay, high data rate and correct jitter [15].

There are different packet scheduling algorithms which are used in LTE-A: Round Robin, Best CQI Scheduler, and Proportional Fair and more where the researchers every day seek to enhance the performance of LTE networks [16]. PS is used to allocate resource blocks for users depending on their Qos requirements and channel condition [17].

\section{Packet Scheduling Algorithms}

In LTE-A, there are many types of PS algorithms. The most famous and powerful algorithms are PF and a best channel quality indicator (Best CQI) [11]. The choice of the suitable PS is an essential design issue in LTE-A and should be based on the traffic types in the network. Moreover, between all PSs there is a trade off in their performance 
between throughput and the other performance indicators: fairness, spectral efficiency and used energy per bit.

PF aims to maximize fairness between users with an agreeable performance in the other performance indicators: average throughput, spectral efficiency and average system energy [11]. Best CQI is a channel-aware scheduling algorithm. It assigns RB to the user which has the best channel quality [11]. This scheduler aims to maximize the average throughput and spectral efficiency while giving an acceptable value of fairness.

\subsection{Proportional Fair Algorithm}

$\mathrm{PF}$ is used in wireless communications. It is the most powerful and efficient algorithm used in LTE-A [11, 12]. Its priority function is shown in Eq. (1) $[18,19] . \mathrm{K}^{*}$ the priority value or each user which used to assign resource blocks for users.

$\mathrm{K}^{*}$ is calculated using the following equation $[18,19,20]$ :

$$
K^{*}=\arg \max _{k}\left(\frac{r_{k, n}}{\left(t_{c}-1\right) T_{k}+\sum_{n=1}^{N} P_{k, n} r_{k, n}}\right)
$$

Where,

$\mathrm{r}_{\mathrm{k}, \mathrm{n}} \quad$ Service rate of $\mathrm{k}_{\mathrm{th}}$ user on the $\mathrm{n}_{\text {th }}$ Resource Block (RB)

$\mathrm{P}_{\mathrm{k}, \mathrm{n}} \quad$ Assignment indicator variable $\left(\mathrm{P}_{\mathrm{k}, \mathrm{n}}=1\right.$, if $\mathrm{n}_{\text {th }} \mathrm{RB}$ is assigned to $\mathrm{k}_{\text {th }}$ user and $=0$ if it's not)

tc Average window size

$\mathrm{T}_{\mathrm{k}} \quad$ Average throughput information of $\mathrm{k}_{\mathrm{th}}$ user. It is computed by equation (2)

$\mathrm{k} \quad$ User index

$\mathrm{n} \quad$ Resource Block index

$$
T_{k}(t+1)= \begin{cases}\left(1-\frac{1}{t_{c}}\right) T_{k, n}(t)+\frac{1}{t_{c}} R_{k}(t), & \mathbf{\kappa}=\mathbf{K}^{*} \\ \left(1-\frac{1}{t_{c}}\right) T_{k, n}(t), & \mathbf{\kappa} \neq \mathbf{K}^{*}\end{cases}
$$

Where,

$\mathrm{T}_{\mathrm{k}} \quad$ Previous average throughput $\mathrm{k}_{\mathrm{th}}$, which assigned to UE in its all previous TTI

$\mathrm{R}_{\mathrm{k}} \quad$ Throughput that UE gets in that TTI

$\mathrm{k} \quad$ User index

$\mathrm{k}=\mathrm{K}^{*}$ when the $\mathrm{k}_{\mathrm{th}}$ user gets resources in the previous TTI. 
Average throughput for UEs is the main factor in the PF priority function. It is computed from the history of Ues used throughput. Therefore, the overall performance of the network is affected by the methods used to compute the average throughput.

\subsection{2 Best CQI Algorithm}

It belongs to channel-aware algorithms. It uses a channel indicator that is carrying the information on how good/bad the communication channel quality is in order to get the RB allocation for each time, without any care about fairness. Best CQI maximizes the throughput by selecting the user with the highest CQI value as shown in Eq. (3) [11].

$$
M_{k, n}=\arg \max _{k}\left(C Q I_{k, n}\right)
$$

Where,

$\mathrm{M}_{\mathrm{k}, \mathrm{n}} \quad$ Maximum value of channel quality indicator

CQI $k$ Channel quality indicator value

$\mathrm{k}^{\text {th }} \quad$ User index

$\mathrm{n}^{\text {th }} \quad$ Resource Block index

CQI sends from each UE to the eNodeB to inform it with its current channel quality Fig. 3 depicts the flow chart of Best CQI scheduler [11]. It creates a 2D array with row represents a UE and column represents assigned RB to each UE. It then chooses the largest element to each UE on a specific RB. After that, it sets the element of the corresponding value of the row $i$ and column $j$ of array element equals 0 . These steps are repeated for all users.

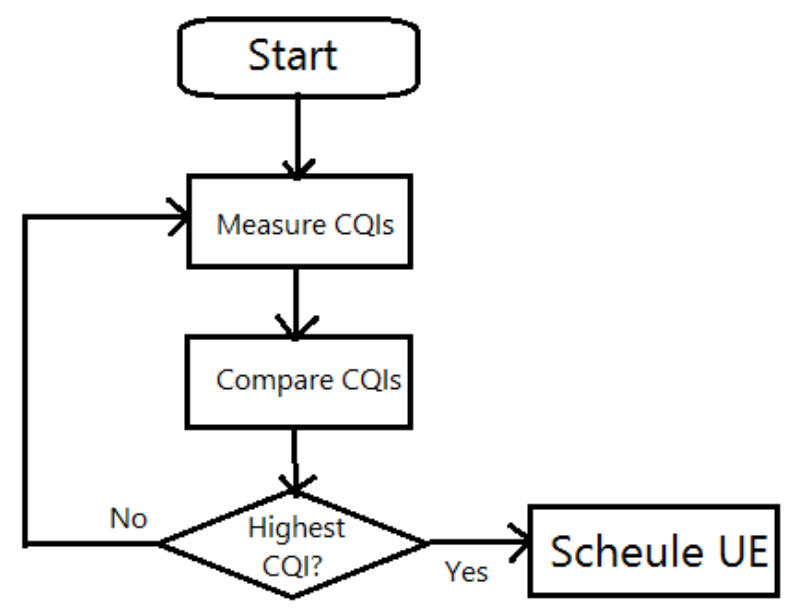

Fig. 3. Flowchart of Best CQI scheduler algorithm. 


\section{$4 \quad$ Proposed Scheduling Algorithm}

The role of PS is allocating of resources in each TTI to UEs to achieve maximization in network performance. In LTE-A, the most famous and powerful algorithm is Proportional Fair (PF) [18]. Many modifications in the PF priority function are introduced in the literature by using different methods to find the average UE throughput.

Some PF modifications are: Midrange Fair Mean, Arithmetic Mean, Median, Midrange Mean, Geometric Mean and Range. These modifications of PF are used for homogeneous LTE-A [4,5].

In this paper, PF modifications performance evaluation is presented for HetNet in LTE-A and a scheduling algorithm is proposed by modifying PF cost function to calculate the average throughput using the root mean square (RMS) method and it is named: Quadratic Proportional Fair (QPF).

To calculate the average for a set of numbers, RMS can be used. It is finding the square root of the average squared values of these numbers. It has the same value as or a little bit larger than the average values which computed by the other used methods. RMS increases the associated resource block per UE. Hence, the total cell throughput and overall network performance are improved.

QPF priority function equation is shown in (4) $T_{k}$,

$$
\mathrm{T}_{\mathrm{k}}(\mathrm{N}+1)=\left(\frac{R_{k}^{2}(1)+R_{k}^{2}(2)+\cdots \ldots \ldots+R_{k}^{2}(N)}{N}\right)^{1 / 2}
$$

Where,

$R_{k}(1), R_{k}(2), \ldots \ldots \ldots \ldots, R_{k}(N)$, is the historical used throughput for $k_{t h} U E$ and $N$ is the number of recently computed throughput values for UE.

This proposed method is compared with other PF modifications which used Arithmetic Mean and Geometric Mean as depicted in [10, 11].

\section{$5 \quad$ Simulation networks and results}

The proposed algorithm is implemented using Vienna LTE System Level Simulator [21]. The performance evaluation of QPF is to prove that it is able to support strongly the QoS with different users' requirements. The impact of user mobility is used in our performance study. QPF is compared with the original PF, Best CQI, PF with arithmetic mean (APF) and PF with geometric mean (GPF) algorithms with various UE velocities for LTE-A HetNet.

LTE-A heterogeneous networks used in QPF performance evaluation with some performance indicators: Average throughput, cell throughput, spectral efficiency, energy per bit and Fairness. The simulation parameters are listed in Table 1 [14]. 
Table 1. Simulation Parameters

\begin{tabular}{|c|c|}
\hline Topology & $\begin{array}{c}\text { Hexagonal grid, 26 cells, 3 sectors } \\
\text { per BS, and 1 femto per sector }\end{array}$ \\
\hline Carrier Frequency & $2 \mathrm{GHz}$ \\
\hline Bandwidth & $20 \mathrm{MHz}$ \\
\hline Duplexing & FDD \\
\hline No. of transmitter & 2 \\
\hline No. of receiver & 2 \\
\hline Transmission mode & CLSM \\
\hline BS height & $20 \mathrm{~m}$ \\
\hline Antenna Gain & $15 \mathrm{dBi}$ \\
\hline Receiver height & $1.5 \mathrm{~m}$ \\
\hline BS power & $45 \mathrm{dBm}$ \\
\hline Femto power & $30 \mathrm{dBm}$ \\
\hline Number of UEs per femto & 10 \\
\hline Number of UEs per BS & 20 \\
\hline User distribution in site area & Uniform \\
\hline Simulation time & $10 \mathrm{TTI}$ \\
\hline
\end{tabular}

\subsection{Network layout}

An LTE-A heterogeneous network with hexagonal geometry is used. The used network layout contains 26 Tri-sector antennas BSs and one femto BS for each sector with $500 \mathrm{~m}$ distance between the BSs. Fig. 4 illustrates the simulated network layout.

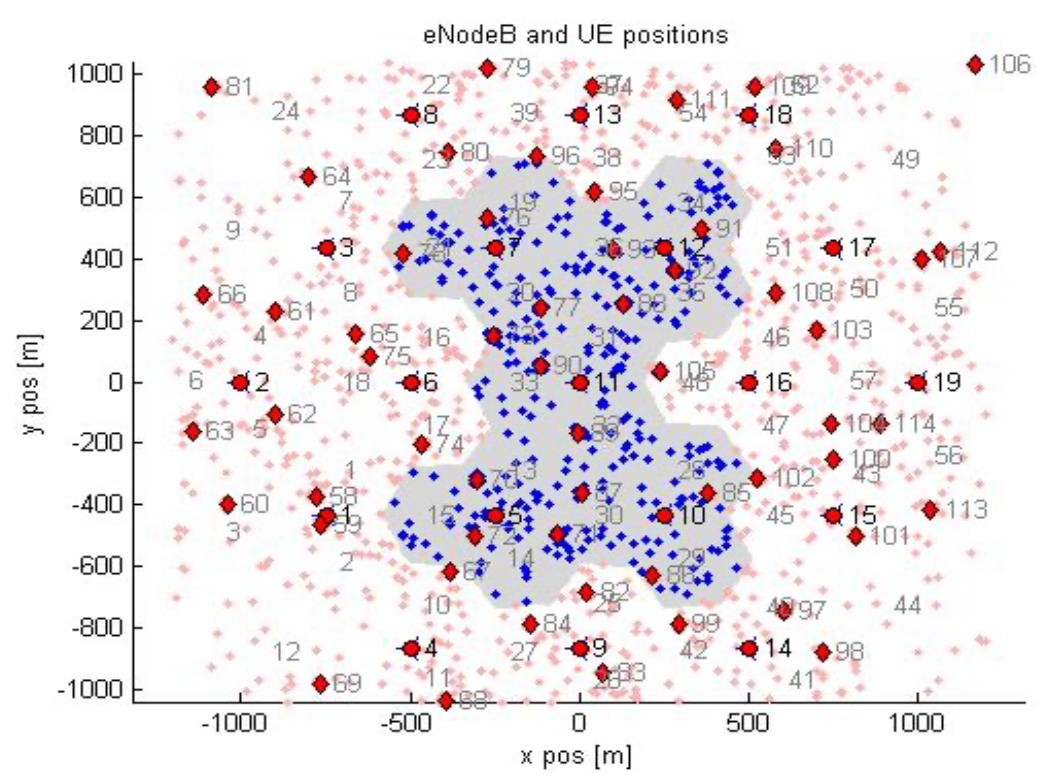

Fig. 4. Simulated Network Layout 


\subsection{Results Analysis}

In this paper, the performance evaluation of QPF is introduced by comparing its performance with the most used algorithms in LTE-A networks: the original PF, Best CQI, PF with arithmetic mean (AMPF) and PF with geometric mean (GMPF) with the impact of user velocity. UEs speed is considered from low speed $3 \mathrm{Km} / \mathrm{h}$, through medium speed $60 \mathrm{Km} / \mathrm{h}$ and finally, high speed $120 \mathrm{Km} / \mathrm{h}$.

Average UE throughput is shown in fig.5 at different UEs speed: 3, 60, and 120 $\mathrm{Km} / \mathrm{h}$. As the results illustrate, Best CQI has the largest throughput at low and medium speeds. But, in high speed QPF has the best throughput value. This is due to that the channel status can be poor with high UEs velocity and it is also caused by using the RMS value to compute the average throughput in QPF, which gives an average value greater than the average value calculated by other methods (PF, Arithmetic Mean Method which used in (AMPF) and Geometric Mean Method which used in (GMPF)).

The average UE Spectral efficiency is depicted in fig. 6. It also experiences decreasing when the velocity increases in all algorithms. However, QPF exceeds all algorithms in spectral efficiency because spectral efficiency is functioning in average throughput. QPF uses RMS to compute the average throughput to achieve a large value of average throughput.

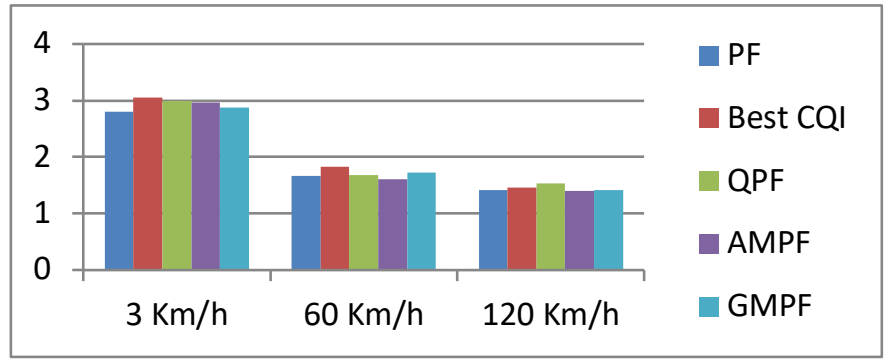

Fig. 5. Average UE Throughput (Mbps) vs. UEs Speed (Km/h)

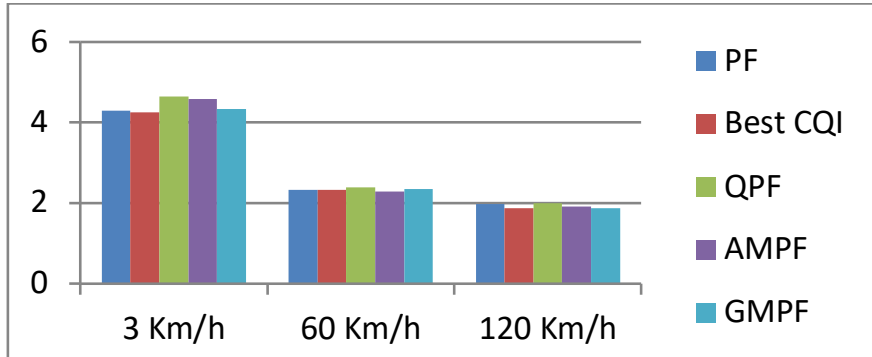

Fig. 6. Average UE Spectral Efficiency (bits/Hz) vs. UEs Speed $(\mathrm{Km} / \mathrm{h})$

Average Energy per bit is shown in fig. 7. As clearly shown, QPF has the least average energy per bit in all velocities. QPF also outperforms the others in energy use. As clearly shown in Fig. 8, Best CQI has the best value of cell throughput at low and 
medium velocities. However, in high UE velocity QPF exceeds all algorithms. The increasing of the average cell throughput in QPF is caused by using of the RMS with all UEs speeds.

Fairness performance indicator is shown in fig. 9. As the figure clearly depicts, QPF and AMPF have better value of fairness than the others in all UEs speeds.

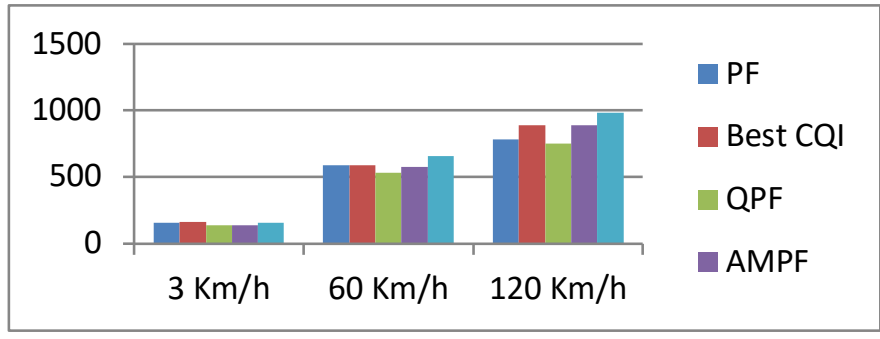

Fig. 7. Average Energy per bit (J) vs. UEs Speed $(\mathrm{Km} / \mathrm{h})$

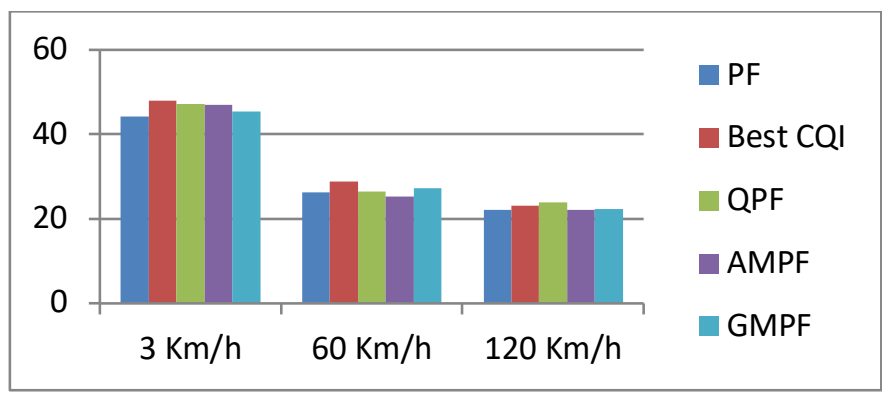

Fig. 8. Average cell Throughput (Mbps) vs. UEs Speed $(\mathrm{Km} / \mathrm{h})$

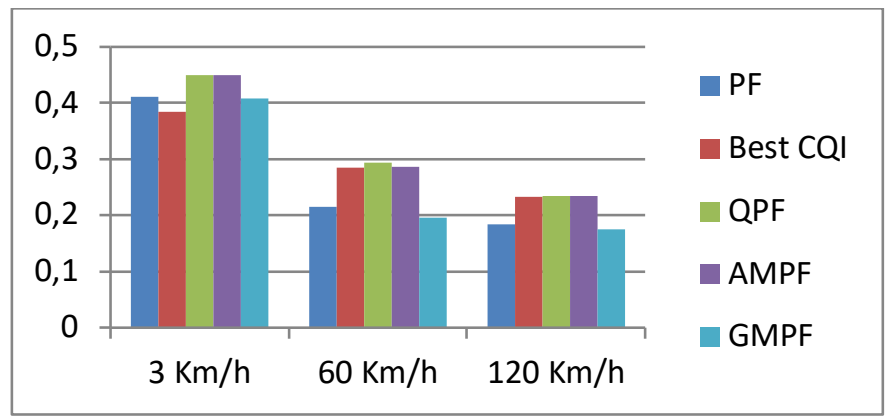

Fig. 9. Fairness vs. UEs Speed $(\mathrm{Km} / \mathrm{h})$

As illustrated from the previous performance evaluation of the algorithms. QPF has best improved for spectral efficiency, energy per bit and fairness by $8.4 \%, 14 \%$, and $9.3 \%$, respectively than original PF. However, Best CQI has the best average UE and cell throughput than all algorithms by $2 \%$ and $1.8 \%$ in low and medium UE velocity, 
respectively. But, QPF has the best value of all types of throughput at high speed. QPF and GMPF has the same performance in fairness with all UEs speeds.

\section{Conclusion and Future Works}

The new mobile communication technologies offer many solutions for assuring QoS for the advanced real-time applications. Strong cell recovery is needed also. So, there is a persistent need for wireless broadband technologies such as LTE-A which can strongly support QoS by selecting a reliable and powerful packet scheduling algorithm and introduce good cell coverage by using of heterogeneous capability.

In this paper, modifications of PF algorithm are proposed, by using different methods for computing the average throughput value which is the main and important parameter in the PF cost function. These methods are the root mean square, arithmetic mean, and geometric mean which is used in LTE-A homogenous networks in the literature $[9,10]$. Complete performance evaluation of the proposed algorithms is introduced by comparing their performance with the original PF and Best CQI algorithms with various UE velocities. The performance indicators are average UE throughput, spectral efficiency, energy per bit, cell throughput and fairness.

The results reveal that QPF has best improved spectral efficiency, energy per bit and fairness by $8.4 \%, 14 \%$, and $9.3 \%$, respectively than original PF. However, Best CQI has a better value of average UE and cell throughput than all algorithms with an improvement of $2 \%$ and $1.8 \%$ in low and medium UE velocity, respectively, but QPF has the best value of all types of throughput at high speed. QPF and GMPF have the same performance in fairness with all UEs speeds. The evaluation of the proposed algorithm in $5 \mathrm{G}$ communication technology with the effective of admission control is considered as our future works.

\section{$7 \quad$ References}

[1] G.Enzo,L.James and Z.Yang, "The Performance Analysis of LTE Network", Communication Networks Spring, 2014.

[2] T. Girici,C. Zhu, J. R. Agre, and A. Ephremides"Proportional Fair Scheduling Algorithm in OFDMA-Based Wireless Systems with QoS Constraints", Journal of Communications and Networks, Vol. 12, No. 1, FEB. 2010. https://doi.org/10.1109/jen.2010.6388432

[3] A.Khanderkar , et.al, "LTE-Advanced: Heterogeneous Networks", European Wireless Conference, San Diego, 2010.

[4] S. Fouziya Sulthana and R. Nakkeeran, "Study of Downlink Scheduling Algorithms in LTE Networks", Journal of Networks, Vol. 9, No. 12, Dec. 2014. https://doi.org/10.4304/ jnw.9.12.3381-3391

[5] S.Schwarz,C. Mehlführer and M. Rupp "Throughput Maximizing Multiuser Scheduling with Adjustable Fairness", Proceedings of the IEEE International Conference on Communications (ICC), pp. 1-5, June 2011. https://doi.org/10.1109/icc.2011.5963489

[6] A. B. Shams, S. R. Abied, Md. Asaduzzaman and Md. F. Hossain," Mobility Effect on the Downlink Performance of Spatial Multiplexing Techniques under Different Scheduling Algorithms in Heterogeneous Network", International Conference on Electrical, Computer 
and Communication Engineering (ECCE), 2017. https://doi.org/10.1109/ecace.2017.7913 $\underline{032}$

[7] A. Rajesh, and R. Achar, "A Review of Heterogeneous Resource Allocation in LTE-A based Femto cell Networks with CQI Feedback", Indian Journal of Science and Technology, Vol. 9, No. 36, 2016. https://doi.org/10.17485/ijst/2016/v9i36/102109

[8] Y. Wang, K. S.garan2, X. Zhu, J. Fei, X. Kong and C. C. Lin," Frequency and Time Domain Packet Scheduling Based on Channel Prediction With Imperfect Cqi In Lte", International Journal of Wireless \& Mobile Networks (IJWMN) Vol. 5, No. 4, August 2013. https://doi.org/10.5121/ijwmn.2013.5412

[9] Nada M. Elshennawy," Quadratic Proportional Fair Scheduling Algorithm for LTE-A Networks", International Journal of Engineering Research and Technology. ISSN 09743154, Volume 12, Number 11, pp. 1957-1963, 2019.

[10] A. Büyükoğlu, M. İzzet Sağlam, A. Kavas, and M. Kartal, "An Efficient Throughput Averaging Method for Proportional Fair Algorithm Used in Mobile Networks," Proceedings of the IEEE Conference on Advances in Wireless and Optical Communications (RTUWO), 2016. https://doi.org/10.1109/rtuwo.2016.7821876

[11] Mai Ali Ibraheem, Nada M. El-Shennawy, A Sarhan, “A Proposed Modified Proportional Fairness Scheduling (MPF-BCQI) Algorithm with Best CQI Consideration for LTE-A Networks", 13th International Conference Computer Engineering and Systems (ICCES), pp. 360-368, Cairo, Egypt, 2018. https://doi.org/10.1109/icces.2018.8639213

[12] M. W. Akhtar,R. Ghaffar, I. Rashid," A Q Learning and Fuzzy Q Learning Approach for Optimization of Interference Constellations in Femto-Macro Cellular Architecture in Downlink",Springer Science+Business Media, New York ,2016. https://doi.org/10.1007/ s11277-016-3206-Z

[13] F. Capozzi, G. Piro, LA .Grieco, G. Boggia and P. Camarda, "Downlink Packet scheduling in LTE cellular networks: Key design issues and a survey", IEEE Transaction on Vehicular Technoly, Vol. 15, No. 2, pp. 678-700, 2012. https://doi.org/10.1109/surv.2012.060912. $\underline{00100}$

[14] S.V. George, L. Mathews and S.S. Pillai, "Physical Layer Frame Structure in 4G LTE/LTEA Downlinkbased on LTE System Toolbox", IOSR Journal of Electronics and Communication Engineering (IOSR-JECE) ,Vol. 10, Issue 3, pp. 12-16, 2015. https://doi. org/10.9790/2834-1203025153

[15] A. Khandekar, N. Bhushan, J. Tingfang and V. Vanghi, " LTE-Advanced: Heterogeneous Networks ", European Wireless Conference, Italy, 2010. https://doi.org/10.1109/ew.2010. $\underline{5483516}$

[16] Y. Wang, K. S.garan2, X. Zhu, J. Fei, X. Kong and C. C. Lin," Frequency and Time Domain Packet Scheduling Based on Channel Prediction With Imperfect Cqi In Lte", International Journal of Wireless \& Mobile Networks (IJWMN) Vol. 5, No. 4, August 2013. https://doi.org/10.5121/ijwmn.2013.5412

[17] A. Ebrahim ,E. Alsusa, and M. W. Baidas," An Uncoordinated Frequency Allocation Scheme for Future Femtocell Networks ", IEEE ,School of Electrical and Electronic Engineering, University of Manchester, 2016. https://doi.org/10.1109/iwcmc.2016.7577 $\underline{064}$

[18] F. Capozzi, G. Piro, LA .Grieco, G. Boggia and P. Camarda, "Downlink Packet scheduling in LTE cellular networks: Key design issues and a survey", IEEE Transaction on Vehicular Technoly, Vol. 15, No. 2, pp. 678-700, 2012. https://doi.org/10.1109/surv.2012.060912. $\underline{00100}$ 
[19] R. Kwan, C. Leung, and J. Zhang, "Proportional fair multiuser scheduling in LTE,” IEEE, Signal Processing Letters, vol. 16, pp. 461 -464, June 2009. https://doi.org/10.1109/lsp. $\underline{2009.2016449}$

[20] M. K. Ismail et. al., "Design and Analysis of Modified-Proportional Fair Scheduler for LTE Femtocell Networks," Journal of Telecommunication, Electronic and Computer Engineering, 2017.

[21] M. Rupp, S. Schwarz and M. Taranetz, "TheVienna LTE-Advanced Simulators Up and Downlink, Link and System Level Simulation", Springer Science+Business Media, Singapore 2016. https://doi.org/10.1007/978-981-10-0617-3

\section{Author}

Nada M. Elshennawy, is an Assistanat Professor, Faculty of Engineering at Tanta University, Tanta in Egypt.

Article submitted 2020-03-16. Resubmitted 2020-04-13. Final acceptance 2020-04-13. Final version published as submitted by the authors. 